\title{
Factores de riesgo asociados a la hernia inguinal recurrente en el adulto mayor
}

\author{
Fernando Karel Fonseca S. ${ }^{1}$, Andrés Lorenzo M. ${ }^{1}$, \\ Yaima Susana Rey V. ${ }^{1}$ y Rolando Llópiz S. ${ }^{1}$
}

\section{Risk factors associated with recurrent inguinal hernia in the elderly}

Objective: To evaluate the association between some risk factors and recurrent inguinal hernia in the elderly. Materials and Method: An analytical case-control study was conducted in the 2002-2011 decade at the "Celia Sánchez Manduley" Hospital, of Manzanillo, Cuba. Initially, a univariate analysis was performed, factors that were statistically significant in the latter were chosen for the subsequent multivariate analysis. Results: In the univariate analysis, 11 variables were associated with inguinal hernia recurrence, except diabetes mellitus $(p=0.051)$. In the multivariate analysis a more adjusted model was obtained with the following variables: delay greater than or equal to 3 years for the first repair $(p=0.00)$, urgent surgery $(p=0.00)$ and sliding of the primary hernia $(p=0.00)$. Discussion: The delay in surgery implies that the patient is getting older and the hernia complex, increasing the risk of suffering from chronic diseases that aggravate the prognosis giving rise to hernia recurrence. On the other hand, the risks involved in carrying out surgery in complicated inguinal hernia: increased surgical time, edema and swelling of the musclefascio-aponeurotic complex, as well as the risk of infection of the operative site. In inguinal hernias slipped recurrences are more frequent, probably due to the high degree of complexity from the point of view of their repair. Conclusion: The delay greater than or equal to 3 years for the first repair, urgent surgery and the sliding of the primary hernia were associated significantly to recurrent inguinal hernia in the elderly Key words: inguinal hernia; recurrence; associated factors.

\section{Resumen}

Objetivo: Evaluar la asociación entre algunos factores de riesgo y la hernia inguinal recurrente en el adulto mayor. Materiales y Método: Se realizó un estudio analítico de casos y controles en el decenio 2002-2011 en el Hospital "Celia Sánchez Manduley", de Manzanillo, Cuba. Se realizó inicialmente un análisis univariado, los factores que resultaron estadísticamente significativos en este último fueron escogidos para el análisis multivariado posterior. Resultados: En el análisis univariado se asociaron 11 variables a la recurrencia herniaria inguinal, excepto la Diabetes mellitus $(p=0,051)$. En el análisis multivariado se obtuvo un modelo más ajustado con las siguientes variables: demora mayor o igual a 3 años para la primera reparación $(\mathrm{p}=0,00)$, cirugía urgente $(\mathrm{p}=0,00)$ y deslizamiento de la hernia primaria $(\mathrm{p}=0,00)$. Discusión: La demora en la cirugía implica que el paciente sea cada día más añoso y la hernia más compleja, aumentando el riesgo de padecer enfermedades crónicas que agravan el pronóstico dando lugar a la recurrencia herniaria. Por otro lado, los riesgos que implica llevar a cabo la cirugía en la hernia inguinal complicada: aumento del tiempo quirúrgico, edema y tumefacción del complejo músculo-fascioaponeurótico, así como por el riesgo de infección del sitio operatorio. En las hernias inguinales deslizadas las recidivas son más frecuentes, debiéndose probablemente al alto grado de complejidad desde el punto de vista de su reparación. Conclusión: La demora mayor o igual a 3 años para la primera reparación, la cirugía urgente y el deslizamiento de la hernia primaria se asociaron significativamente a la hernia inguinal recurrente en el adulto mayor.

Palabras clave: hernia inguinal; recurrencia; factores asociados.
'Cátedra de Cirugía General Hospital Provincial ClínicoQuirúrgico Docente "Celia Sánchez Manduley". Manzanillo. Granma. Cuba.

Recibido el 14 de abril de 2018 y aceptado para publicación el 30 de mayo de 2018.

Correspondencia a: Dr. Fernando Karel F. ffonsecasosa@gmail.com 


\section{Introducción}

La recurrencia es la complicación más frecuente a largo plazo en herniorrafia inguinal. La introducción de las técnicas "tensión-free" utilizando mallas y de la cirugía laparoscópica de las hernias ha contribuido a bajar estas tasas, pero, a pesar de estos adelantos, las hernias inguinales recidivadas constituyen aún una elevada proporción de las herniorrafias inguinales, con escasa tendencia a disminuir ${ }^{1}$.

La definición de recurrencia se asume como la reaparición de un defecto herniario en el mismo sitio anatómico específico de la hernia primariamente operada después de una desaparición quirúrgica temporal; de otro modo, se tratará de una segunda hernia primaria y diferente ${ }^{2}$.

Si se ha aplicado una técnica de reparación libre de tensión con reforzamiento preventivo de otras zonas vulnerables, mediante material protésico y reaparece otro defecto en un sitio diferente al primariamente operado, se denominará reparación inguinal profiláctica fallida con defecto residual en la región inguinal ${ }^{2}$.

Existen varios factores de riesgo asociados a la presentación de esta patología, como son la edad mayor de 60 años, el sobrepeso, la uropatía obstructiva, el estreñimiento, la tos crónica, las colagenopatías, entre otros; la mayoría de estos persisten tras la reparación herniaria, por lo que la probabilidad de recidiva y/o de hernia contralateral se mantiene elevada en el tiempo ${ }^{3}$.

El objetivo de esta investigación será evaluar la asociación entre algunos factores de riesgo y la hernia inguinal recurrente en el adulto mayor, la incorporación de tales conocimientos permitirá un enfoque y abordaje más objetivo y actual del proceso de atención en salud que se dispensa a este grupo; ello, eventualmente, permitiría el diseño e implementación de acciones específicas con el fin de mejorarlo.

\section{Materiales y Métodos}

\section{Diseño de estudio}

Se realizó un estudio observacional, analítico, de casos y controles hospitalarios en el Hospital Clínico Quirúrgico Docente "Celia Sánchez Manduley", de Manzanillo, Granma, Cuba.

Período de estudio: enero de 2002 a diciembre de 2011.

Población hospitalaria: 978 pacientes con 65 años o más intervenidos quirúrgicamente por hernia inguinal.

\section{Criterios de inclusión}

Casos: 103 pacientes intervenidos quirúrgicamente por hernia inguinal recurrente.

Controles: 206 pacientes intervenidos quirúrgicamente por hernia inguinal no recurrente o primaria; se seleccionaron 2 controles por caso teniendo en cuenta criterios de pareamiento por género.

\section{Criterios de exclusión}

Se excluyeron 4 casos cuyos expedientes clínicos e informes operatorios no contenían los datos necesarios para la investigación.

Variable dependiente: Hernia inguinal recurrente.

Variables independientes: Variables clínicas (hernia primaria indirecta, hábito de fumar, hernia lado derecho, diabetes mellitus, obesidad, demora mayor o igual a 3 años para la primera reparación), variables quirúrgicas (herniorrafia, cirugía urgente, tiempo quirúrgico igual o mayor de 90 minutos, deslizamiento de la hernia primaria, residente, infección del sitio operatorio).

La información se obtuvo a partir de fuentes secundarias, representadas por los expedientes clínicos e informes operatorios de cada paciente, identificados en el departamento de admisión, archivo y estadísticas de este centro. La recolección de los datos estuvo a cargo del propio autor, mediante una planilla de recolección de datos. Estos últimos fueron introducidos y procesados en el programa IBM SPSS Statistics para Windows ver. 24.0.

Se realizó, inicialmente, un análisis univariado para asociar los diferentes factores de riesgo a la recurrencia herniaria, que se basó en la estimación de los Odds Ratio (OR). Se identificaron como factores de riesgo asociados aquellas variables independientes que luego de ser analizadas sus OR fueron superiores al valor 1 .

La verificación de la existencia de la asociación significativa (no debida al azar) se realizó mediante la prueba de $\chi^{2}$ (Ji Cuadrado) y la construcción de intervalos de confianza del $95 \%$ para cada OR.

Se consideraron estadísticamente significativas cuando los valores $\mathrm{p}$ (nivel de significación) fueron menores que $0,05 \mathrm{y}$ el intervalo de confianza no incluyó al valor 1 .

Los factores de riesgo que resultaron estadísticamente significativos en el análisis univariado fueron escogidos para el análisis multivariado posterior, que se realizó mediante la regresión logística múltiple (RLM) por el método de Wald hacia adelante, a fin de obtener una estimación estandarizada de los ORy controlar las variables confusoras. Los resultados fueron presentados en cuadros estadísticos.

En esta investigación se tuvo en cuenta los 
principios básicos de la bioética médica tales como autonomía o respeto por las personas, beneficencia, no maleficencia y justicia.

\section{Resultados}

\section{Análisis univariado}

El análisis univariado de la asociación entre diferentes variables clínicas y el riesgo de recurrencia herniaria se mostró en la Tabla 1.

La hernia primaria indirecta $(p=0,010)$, el hábito de fumar $(\mathrm{p}=0,000)$, hernia en el lado derecho $(\mathrm{p}=0,000)$, obesidad $(\mathrm{p}=0,009)$, y la demora mayor o igual a 3 años para la primera reparación $(\mathrm{p}=0,000)$ se asociaron significativamente a la recurrencia de hernia. Por otro lado, la diabetes mellitus se consideró un factor de riesgo en nuestro estudio $(\mathrm{OR}=2,71)$, pero este valor no se consideró significativo estadísticamente $(\mathrm{p}=0,051)$ por lo que se excluyó del análisis posterior.

La Tabla 2 detalla el análisis univariado de la asociación entre diferentes variables quirúrgicas y el riesgo de recurrencia herniaria, en ella se observó que todas las variables quirúrgicas se asociaron significativamente a la presencia de hernia inguinal recurrente.

\section{Análisis multivariado.}

Se incluyeron las 11 variables resultantes de los análisis univariados previos. Se obtuvieron 8 variables con resultados no significativos $(p>0,05)$, por lo que no se consideraron los valores de sus OR. Estas variables fueron: hernia primaria indirecta, hábito de fumar, hernia lado derecho, obesidad, herniorrafia, tiempo quirúrgico igual o mayor de 90 minutos, residente, infección del sitio operatorio.

El modelo más ajustado (Tabla 3 ) quedó constituido por las variables cuyos OR se mencionan a continuación. Los OR más altos lo mostraron la demora mayor o igual a 3 años parala primera reparacióncon 16,75 , lo que significa que es casi 17 veces más probable que aparezca la recurrencia herniaria en los adultos mayores con demora mayor o igual a 3 años parala primera reparaciónque en los que la demora fuese menor de 3 años. En segundo

Tabla 1. Análisis univariado de la asociación entre diferentes variables clínicas y el riesgo de recurrencia herniaria

\begin{tabular}{|c|c|c|c|c|c|}
\hline Variables clínicas & $\begin{array}{c}\text { Casos } \\
n=103\end{array}$ & $\begin{array}{c}\text { Controles } \\
\mathbf{n}=\mathbf{2 0 6}\end{array}$ & OR $^{\mathbf{a}}$ & IC $95 \%$ & $\mathbf{p}$ \\
\hline Hernia primaria indirecta & 67 & 102 & 1,89 & $(1,35-2,61)$ & 0,010 \\
\hline Hábito de fumar & 52 & 50 & 3,18 & $(1,95-5,20)$ & 0,000 \\
\hline Hernia lado derecho & 87 & 102 & 5,54 & $(3,36-9,11)$ & 0,000 \\
\hline Diabetes mellitus & 9 & 7 & 2,71 & $(0,75-9,70)$ & 0,051 \\
\hline
\end{tabular}

${ }^{\mathrm{a}}$ Odds Ratio. ${ }^{\mathrm{b}}$ Intervalo de confianza del 95\%.

Tabla 2. Análisis univariado de la asociación entre diferentes variables quirúrgicas y el riesgo de recurrencia

\begin{tabular}{|c|c|c|c|c|c|}
\hline Variables quirúrgicas & $\begin{array}{c}\text { Casos } \\
n=103\end{array}$ & $\begin{array}{c}\text { Controles } \\
\mathbf{n}=\mathbf{2 0 6}\end{array}$ & $\mathbf{O R}^{\mathbf{a}}$ & IC $95 \%$ & $\mathbf{p}$ \\
\hline Herniorrafia & 78 & 70 & 6,06 & $(3,60-10,26)$ & 0,000 \\
\hline Tiempo quirúrgico igual o mayor de 90 minutos & 22 & 18 & 2,83 & $(1,47-5,50)$ & 0,001 \\
\hline Deslizamiento de la hernia primaria & 19 & 9 & 4,95 & $(2,18-11,20)$ & 0,001 \\
\hline Infección del sitio operatorio & 6 & 2 & 6,30 & $(1,27-31,17)$ & 0,011 \\
\hline
\end{tabular}

${ }^{\mathrm{a}}$ Odds Ratio. ${ }^{\mathrm{b}}$ Intervalo de confianza del 95\%. 
Tabla 3. Resultados del análisis multivariado mediante regresión logística múltiple de las variables asociadas a la recurrencia de hernia inguinal

\begin{tabular}{|c|c|c|c|c|}
\hline Variables & Wald $^{\mathrm{a}}$ & $\mathbf{O R}^{\mathbf{b}}$ & IC $95 \%{ }^{c}$ & $\mathbf{p}$ \\
\hline Demora mayor o igual a 3 años para la primera reparación & 39,055 & 16,759 & $6,923-40,569$ & 0,000 \\
\hline Cirugía urgente & 12,997 & 4,184 & $1,564-7,462$ & 0,000 \\
\hline
\end{tabular}

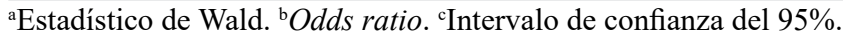

lugar, aparece la cirugía urgente con 4,18, lo que significa que es 4 veces más probable que aparezca recurrencia herniaria en los ancianos que se intervienen quirúrgicamente de urgencia que aquellos que se operan de forma electiva. Finalmente, aparece la variable deslizamiento de la hernia primaria con 3,39 , es decir, es 3 veces más probable que aparezca la recurrencia herniaria en los ancianos con deslizamiento de la hernia primaria con relación a los que no la tuviesen.

\section{Discusión}

La discusión reposa en la comparación de hallazgos entre los estudios aquí presentados y los realizados por otros autores. A ello se adiciona cierto nivel de especulación, a partir de la experiencia y de la bibliografía consultada.

La identificación de los factores de riesgo que influyen en la recurrencia de la hernia inguinal, significa por lo menos, un primer paso hacia la disminución de su prevalencia.

La elección de un tratamiento quirúrgico apropiado es más difícil en la reparación de una hernia inguinal recurrente que en la de una hernia primaria ${ }^{4}$.

Todas las medidas para disminuir el número de recurrencias deberían ser usadas, las cuales solo podrían realizarse obteniendo el conocimiento de factores de riesgo relacionados con el paciente junto con el conocimiento de los factores de riesgo técnicos controlables 5 .

Carbonell F. ${ }^{6}$ menciona que la demora mayor o igual a 3 años para la primera reparación es una causa que puede explicar la aparición de las recurrencias herniarias, coincidiendo con este estudio.

Esto se explica lógicamente porque mientras más tiempo tenga la hernia primaria sin intervención quirúrgica su tamaño va a ser mayor, comprometiendo las estructuras fascio-aponeuróticas, con más posibilidades de recidivar.

La demora en el tratamiento quirúrgico también implica que el paciente sea cada día más viejo y la hernia más compleja, con lo cual aumenta el riesgo de padecer enfermedades crónicas asociadas como las cardiopatías, la obesidad, la diabetes mellitus y otras que agravan el pronóstico del enfermo dando lugar a la recurrencia herniaria.

Numerosos estudios coinciden con el nuestro en que la cirugía de urgencia puede ser un factor de riesgo para la hernia inguinal recurrente $\mathrm{e}^{7-9}$.

Esto se debe a que el periodo preoperatorio mediato prácticamente no existe en la cirugía de urgencia, lapso de tiempo indispensable para tratar de que el enfermo llegue al acto quirúrgico en condiciones óptimas. El paciente desnutrido presentará déficit en las proteínas y se conoce la importancia de estos elementos, no solo en la cicatrización, donde juegan un papel fundamental, sino también en los aspectos inmunológicos, que estarán muy presentes en la respuesta del organismo ante la agresión que significa el acto quirúrgico. Los casos con enfermedad pulmonar obstructiva crónica (EPOC) tienen un mayor riesgo de presentar complicaciones respiratorias posoperatorias y se pueden beneficiar de tratamientos en el preoperatorio mediato. En este periodo, además, se deben de tratar las afecciones genitourinarias sobre todo las prostáticas que se relacionan con la recurrencia hernia.

Por otro lado, todos los riesgos que implica llevar a cabo la cirugía en la hernia inguinal complicada como son: el aumento del tiempo quirúrgico, el edema y tumefacción del complejo músculo-fascioaponeurótico, así como por el riesgo de la infección del sitio operatorio que conllevan al final a la recurrencia herniaria.

Estudios nacionales ${ }^{10,11} \mathrm{e}$ internacionales ${ }^{12-13}$ muestran que la hernia inguinal recurrente es más frecuente en pacientes con deslizamiento de la hernia primaria.

Las hernias inguinales por deslizamiento comprenden el $1,5 \%$ de todas las hernias afectando más al hombre obeso que a la mujer, su relación según la edad es evidentemente una enfermedad de adultos mayores siendo las recidivas posoperatorias más frecuentes que en cualquier otro tipo de hernias, de- 
biéndose probablemente al gran tamaño del orificio herniario, así como al alto grado de complejidad desde el punto de vista de su reparación.

En la hernia inguinal por deslizamiento, el anillo inguinal profundo está muy dilatado por la víscera que contiene y, generalmente, se acompaña de debilidad en la pared posterior del conducto inguinal.

Al realizar el análisis multivariado mediante la regresión logística múltiple, se obtuvo un modelo ajustado del cual quedaron excluidas algunas variables que demostraron una asociación importante en el análisis univariado, tal es el caso de las variables herniorrafia, obesidad y hábito de fumar, cuya relación con la hernia inguinal recurrente está bien argumentada. En tal sentido debe recordarse que los métodos de análisis estadísticos utilizan procedimientos algorítmicos que no distinguen los aspectos teóricos implicados en los mismos. Por tanto, no debe soslayarse la importancia que, basados en la experiencia de tales estudios, se le confiere a cada uno de los factores mencionados.

\section{Conclusión}

La demora mayor o igual a 3 años para la primera reparación, la cirugía urgente y el deslizamiento de la hernia primaria, se asociaron significativamente a la hernia inguinal recurrente en el adulto mayor.

\section{Agradecimientos}

Dr. Carlos Zamora Linares por su aporte en el análisis estadístico del trabajo.

\section{Responsabilidades éticas}

Protección de personas y animales. Los autores declaran que para esta investigación no se han realizado experimentos en seres humanos ni en animales.

Confidencialidad de los datos. Los autores declaran que en este artículo no aparecen datos de pacientes.

Conflictos de interés: ninguno.

\section{Referencias}

1. Acevedo A, León J, García G. Hernia inguinal recidivada. Tratamiento ambulatorio con anestesia local por la vía abierta anterior. Rev Chil Cir. 2015; 67:511-7 [consultado el 21 de febrero de 2018].

2. Cisneros HA. Recurrencia en hernia inguinal. ¿Qué significa? ¿Es un problema semántico? ¿Es problema de lenguaje e interpretación? ¿Es el error del milenio? Cir Gen. 2004;26:260-4. [consultado el 21 de febrero de 2018].

3. Villanueva J, Aguilera A. Intervenciones en hernias inguinocrurales. Bilateralidad y recidiva herniaria. Servicio de Cirugía del Hospital Naval de Viña del Mar. Rev Chil Cir. 2017;69 (3):211-4 [consultado el 21 de febrero de 2018].

4. Pisanu A, Podda M, Saba A, Porceddu G, Uccheddu A. Meta-analysis and review of prospective randomized trials comparing laparoscopic and Lichtenstein techniques in recurrent inguinal hernia repair. Hernia 2015;19:355-66 [consultado el 21 de febrero de 2018].

5. Sharma A, Sarwal A. Surgical repair in recurrent inguinal hernia. Ann Laparosc Endosc Surg. 2017;2:97 DOI 10.21037/ ales.2017.05.03 [consultado el $21 \mathrm{de}$ febrero de 2018].

6. Carbonell F. Hernia inguinocrural. $1^{\text {a }}$ ed. Valencia España: Editorial Ethicon; 2001. p 277

7. The Hernia Surge Group. International guidelines for groin hernia management. In: Jorgensen LN, Hope WW, Bisgaard T, editors. Risk Factors for the Development of Inguinal Hernias in Adults. Hernia. 2018;22(Pt 1):7-8. [consultado el 21 de febrero de 2018].

8. Burcharth J. The epidemiology and risk factors for recurrence after inguinal hernia surgery. Dan Med J. 2014;61:B4846 [consultado el 21 de febrero de 2018].

9. Burcharth J, Pommergaard HC, Bisgaard T, Rosenberg J. Patient-related risk factors for recurrence after inguinal hernia repair: a systematic review and meta-analysis of observational studies. Surg Innov. 2015;22:303-17 [consultado el 21 de febrero de 2018].

10. Abraham JF. Cirugía de las hernias de la pared abdominal. $1^{a}$ ed. Ciudad de la Habana: Editorial Ciencias Médicas; 2010. p 428.

11. Goderich JM, Noguerales F. Hernias de la región inguinocrural. Acerca de su tratamiento quirúrgico. $1^{\text {ra }}$ ed. España: Universidad de Alcalá. Servicio de Publicaciones;1999. p 121 [consultado el 21 de febrero de 2018].

12. Andresen K, Bisgaard T, Rosenberg J. Sliding inguinal hernia is a risk factor for recurrence. Langenbecks Arch Surg. 2015;400:101-6 DOI 10.1007/s00423014-1262-y [consultado el 21 de febrero de 2018].

13. Komorowski AL, Morán-Rodríguez J, Kazi R, Wysocki WM. Sliding inguinal hernias. Int J Surg. 2012;10:206-8 [consultado el 21 de febrero de 2018]. 